Review article

\title{
Linking cognitive and visual perceptual decline in healthy aging: The information degradation hypothesis
}

\author{
Zachary A. Monge ${ }^{\mathrm{a}, *}$, David J. Madden ${ }^{\mathrm{b}}$ \\ a Center for Cognitive Neuroscience, Duke University, Durham, NC 27708, USA \\ ${ }^{\mathrm{b}}$ Brain Imaging and Analysis Center, Duke University Medical Center, Durham, NC 27710, USA
}

\section{A R T I C L E I N F O}

\section{Article history:}

Received 12 May 2016

Received in revised form 25 July 2016

Accepted 26 July 2016

Available online 30 July 2016

\section{Keywords:}

Aging

Cognition

Information degradation

Perception

Vision

Visual perception

\begin{abstract}
A B S T R A C T
Several hypotheses attempt to explain the relation between cognitive and perceptual decline in aging (e.g., common-cause, sensory deprivation, cognitive load on perception, information degradation). Unfortunately, the majority of past studies examining this association have used correlational analyses, not allowing for these hypotheses to be tested sufficiently. This correlational issue is especially relevant for the information degradation hypothesis, which states that degraded perceptual signal inputs, resulting from either age-related neurobiological processes (e.g., retinal degeneration) or experimental manipulations (e.g., reduced visual contrast), lead to errors in perceptual processing, which in turn may affect non-perceptual, higher-order cognitive processes. Even though the majority of studies examining the relation between age-related cognitive and perceptual decline have been correlational, we reviewed several studies demonstrating that visual manipulations affect both younger and older adults' cognitive performance, supporting the information degradation hypothesis and contradicting implications of other hypotheses (e.g., common-cause, sensory deprivation, cognitive load on perception). The reviewed evidence indicates the necessity to further examine the information degradation hypothesis in order to identify mechanisms underlying age-related cognitive decline.
\end{abstract}

(c) 2016 Elsevier Ltd. All rights reserved.

\section{Contents}

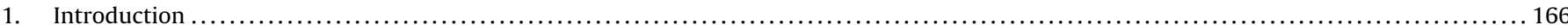

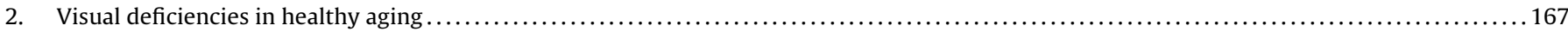

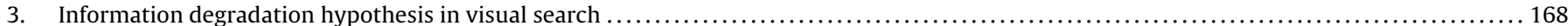

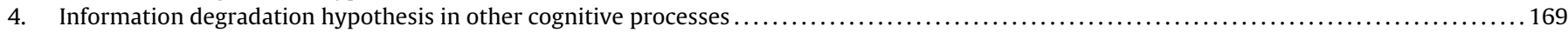

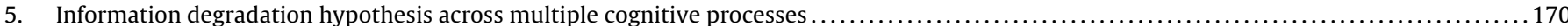

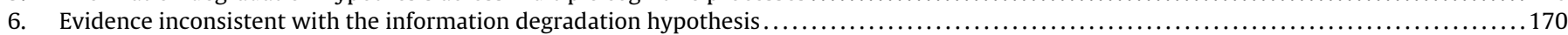

7. Conclusions and future directions . . . . . . .

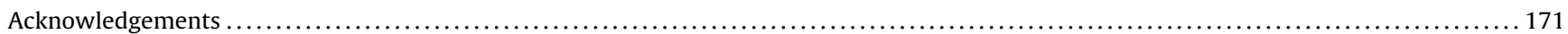

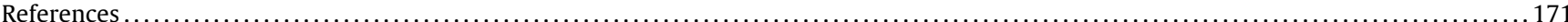

\section{Introduction}

For more than five decades researchers have attempted to study and explain the relation between cognitive and perceptual decline

\footnotetext{
* Corresponding author at: Center for Cognitive Neuroscience, Duke University, Levine Science Research Center, Box 90999 Durham, NC 27708, USA.

E-mail addresses: zachary.monge@duke.edu (Z.A. Monge) david.madden@duke.edu (D.J. Madden).
}

in aging, but this association remains poorly understood. This effort has resulted in four predominant hypotheses within the literature that attempt to explain the relation between age-related cognitive and perceptual decline: (a) the common-cause hypothesis, which states that a third factor associated with aging, which detrimentally affects both cognition and perception, contributes to a decline in these two domains (Baltes and Lindenberger, 1997; Lindenberger and Baltes, 1994); (b) the sensory deprivation hypothesis, which states that over time a decline in perception causes a gradual degradation in neural substrates, which in turn affects cognition (Lindenberger and Baltes, 1994); (c) the cognitive load on perception hypothesis, which states that older adults perform 


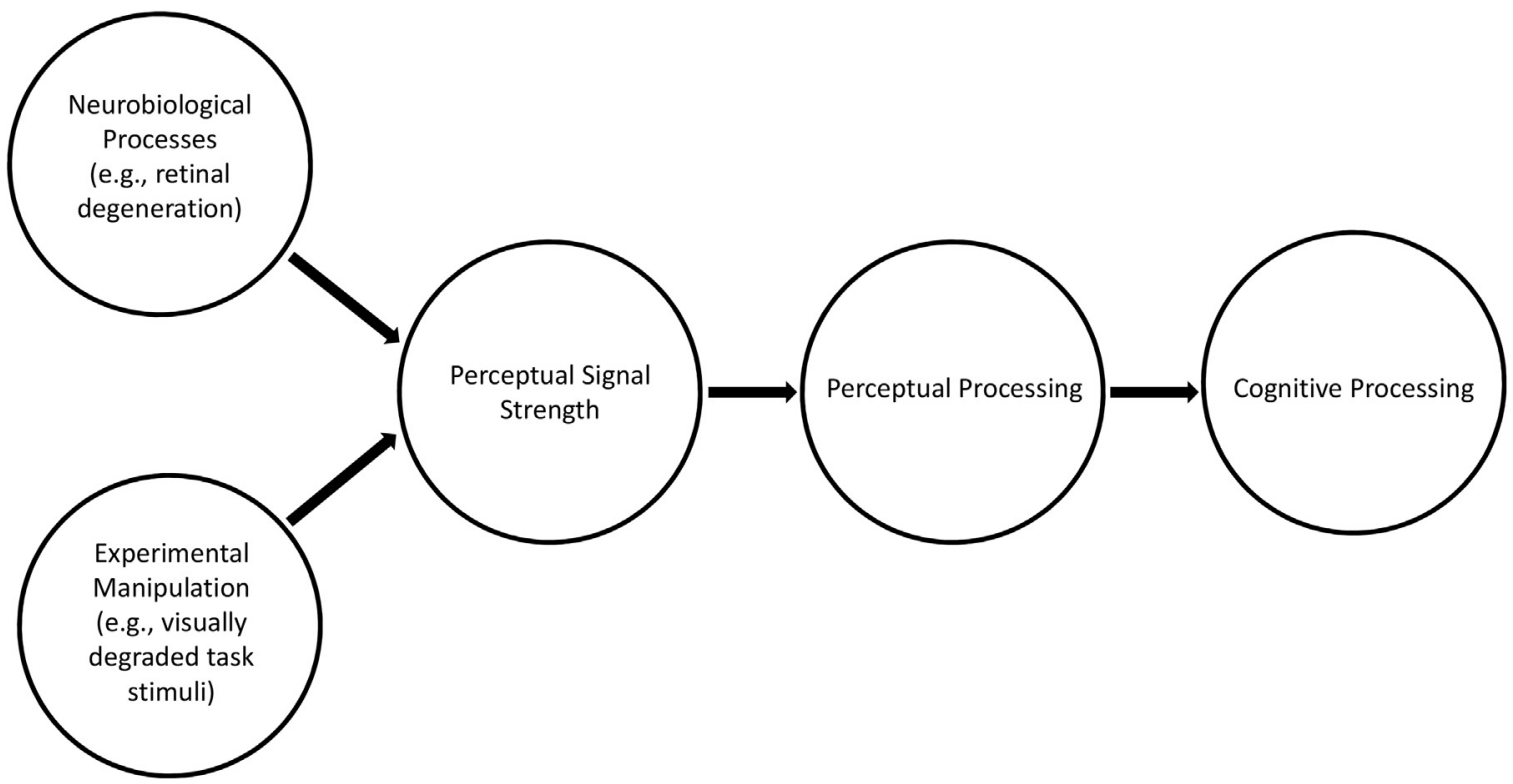

Fig. 1. Schematic of the information degradation hypothesis.

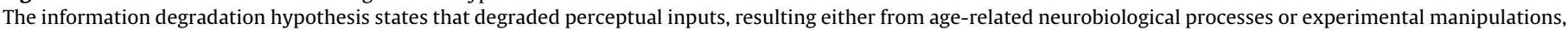
lead to errors in perceptual processing, which in turn may affect non-perceptual, higher-order cognitive processes.

worse on perceptual tasks due to impoverished cognition (Li et al., 2001; Lindenberger et al., 2000), and (d) the information degradation hypothesis, which states that degraded perceptual signal inputs, resulting either from age-related neurobiological processes or experimental manipulations, lead to errors in perceptual processing, which in turn may affect non-perceptual, higher-order cognitive processes (Schneider and Pichora-Fuller, 2000; for a schematic illustration, see Fig. 1).

Unfortunately, most studies examining the association between cognitive and perceptual decline in aging have been correlational (e.g., Baltes and Lindenberger, 1997; Lindenberger and Baltes, 1994), not allowing for these hypotheses to be tested sufficiently. Specifically, correlational analyses cannot determine whether modifying the perceptual signal strength of study participants has immediate effects on cognitive performance; an intervention or experimental manipulation is needed. A demonstration that manipulating perceptual signal strength immediately affects cognitive performance would provide direct support for the information degradation hypothesis, but contradict assumptions of the common-cause, sensory deprivation, and cognitive load on perception hypotheses. The latter hypotheses all imply that modifying the perceptual signal strength of individuals should either not affect or not immediately affect cognitive performance, but each for different reasons. According to the common-cause hypothesis, since a common source independently causes cognitive and perceptual decline, and perception is not hypothesized to be the source, an enhancement of perceptual signal strength should not affect cognition. For the sensory deprivation hypothesis, since this hypothesis states that long-term sensory deprivation leads to neural degeneration and ultimately cognitive decline, it is unlikely that enhancing perceptual signal strength will immediately reverse neural degeneration and enhance cognitive abilities. Lastly, for the cognitive load on perception hypothesis, since it states that a decline in cognition causes a decline in perceptual abilities, enhancing perception should not affect cognition.

Despite the prevalence of correlational analyses examining this relation, several studies have attempted to test the validity of the information degradation hypothesis by experimentally manipulating older and younger adults' perceptual inputs. This hypothesis predicts that both older and younger adults' cognitive performance should be immediately affected by perceptual signal manipulations, but experimental manipulations should have a greater effect on older adults due to (a) age-related slowing of perceptual processing (Faust et al., 1999; Madden, 2001; Salthouse, 1996, 2000) and (b) in some cases insufficient cognitive/neural top-down mechanisms, as a result of age-related neural degeneration (Raz et al., 2005), being unable to compensate for decreased bottom-up, perceptual signals in older adults. In addition, the information degradation hypothesis predicts that different cognitive processes may be differentially affected by an identical bottom-up, perceptual signal manipulation, since individual cognitive states utilize unique top-down mechanisms and bottom-up, perceptual signals may differentially interact with these top-down processes.

Here, we review studies testing the validity of the information degradation hypothesis, specifically studies examining the relation between visual perception and cognition in healthy aging (for a summary of the experimental designs of these studies, see Table 1). Evidence supporting the information degradation hypothesis contradicts aspects of these other three hypotheses (common-cause, sensory deprivation, and cognitive load on perception hypotheses), which imply that cognitive deficiencies in older adults cannot be ameliorated by simply enhancing the visual perceptual signal strength of older adults. Before reviewing these studies, we briefly discuss visual deficiencies associated with healthy aging. Then, we review studies testing the information degradation hypothesis across various cognitive processes. Lastly, we end by proposing future directions for the field. It should be noted that even though the focus of this review is on visual perception, auditory perception also declines with age (e.g., Lin et al., 2014; Lin et al., 2011; Lin et al., 2013) and has previously been associated with principles of the information degradation hypothesis (e.g., Ng et al., 2013; Pichora-Fuller et al., 1995; Tun et al., 2009), but these studies are out of the scope of this brief review. 
Table 1

List of Studies Examining the Information Degradation Hypothesis.

\begin{tabular}{|c|c|c|c|}
\hline Authors (year) & Study Population & Cognitive Task(s) & Experimental Manipulation \\
\hline Toner et al. (2012) & YA, OA, AD, \& PD & Digit cancellation task & Task stimuli contrast \\
\hline Laudate et al. (2012) & $\mathrm{YA}, \mathrm{OA}, \mathrm{AD} \& \mathrm{PD}$ & Bingo task & Task stimuli size and contrast \\
\hline Ben-David and Sneider (2012) & YA & Stroop task & Task stimuli color contrast \\
\hline Gilmore et al. (2006) & YA & Digit-Symbol Substitution Test ${ }^{\mathrm{a}}$ & Task stimuli contrast \\
\hline Dickinson and Rabbitt (1991) & YA & Memory for short passages task & Occlusion filter lenses \\
\hline Anstey et al. (2006a) & $\mathrm{OA}$ & Multiple tasks & Task stimuli contrast \\
\hline Cronin-Golomb et al. (2007) & $\mathrm{YA}, \mathrm{OA}, \& \mathrm{AD}$ & Multiple tasks & Task stimuli contrast \\
\hline Bertone et al. (2007) & YA & Multiple tasks & Occlusion filter lenses \\
\hline Lindenberger et al. (2001) & $\mathrm{OA}$ & Multiple tasks & Occlusion filter lenses \\
\hline Anstey et al. (2006b) & $\mathrm{OA}$ & Multiple tasks & Cataract surgery \\
\hline
\end{tabular}

Note: $\mathrm{AD}=$ Alzheimer's disease patients; $\mathrm{OA}=$ healthy older adults; $\mathrm{PD}=$ Parkinson's disease patients; YA= healthy younger adults.

a Digit-Symbol Substitution Test of the Wechsler Adult Intelligence Scale (WAIS; Wechsler, 1958).

\section{Visual deficiencies in healthy aging}

Healthy aging is associated with a decline in visual perceptual abilities, specifically a decline in (a) visual acuity (Jackson and Owsley, 2003; Owsley, 2011; Owsley et al., 1983), which refers to the ability to detect small, high contrast targets (e.g., the gap in Landolt Cs); and (b) visual contrast sensitivity (Greene and Madden, 1987; Nameda et al., 1989; Owsley et al., 1983; Owsley and Sloane, 1987), which refers to the lowest contrast at which the difference between a homogeneous field and a spatial grating (i.e., an alternating series of light and dark bands) can be detected. Visual acuity decline in aging may at least partially be attributed to increased refractive errors in aging and is further compounded by older adults using expired corrective lens prescriptions (Skeel et al., 2003; Tielsch et al., 1990). Visual contrast sensitivity deficiencies are of particular interest in the study of cognitive aging because (a) contrast sensitivity has been demonstrated to have a stronger association than visual acuity to neuropsychological performance (Skeel et al., 2006); (b) contrast sensitivity is associated with decreased performance in everyday activities, such as driving (Rubin et al., 1994; Wood and Owens, 2005), judging distances and mobility (Rubin et al., 1994), and discriminating highway signs (Evans and Ginsburg, 1985); and (c) recent meta-analyses have failed to find associations between visual acuity and cognitive task performance in aging (Houston et al., 2016; La Fleur and Salthouse, 2014), perhaps indicating that other measures of visual perception, such as contrast sensitivity, may be better indicators of cognition - it should be noted that these meta-analyses only included correlational studies, leading to the possibility that experimental manipulations of visual acuity may affect cognitive performance, as demonstrated in several studies reviewed here.

Several neural changes occur to the visual system in aging that likely account for visual acuity and contrast sensitivity decline (for a review, see Madden et al., 2005). At the level of the sensory organ, there is a general degeneration of the eyes in aging, which decreases the amount of light that may reach the retina in older adults (Weale, 1961). These changes to the eyes include an increase in the density and hardness of the crystalline lens, a decrease in the resting diameter of the pupil, an increase in the opacification of the lens, and a decrease in the number of receptor cells (Scialfa, 2002). At the level of the cortex, during visual perceptual tasks, older compared to younger adults often exhibit decreased magnitude and spatial extent activation of visual sensory cortex (e.g., Buckner et al., 2000; Huettel et al., 2001; Ross et al., 1997; Ward et al., 2015). Visual cortical activation changes in aging may have neural and cognitive consequences, for example, leading older adults to compensate for decreased visual cortex activation by recruiting additional anterior regions (e.g., prefrontal cortex) in service of successful cognition (Davis et al., 2008). However, the mechanisms contributing to decreased visual cortical activation in older adults, and the associated consequences, remain an active area of investigation (Cabeza and Dennis, 2012; Grady, 2012; Park and Reuter-Lorenz, 2009). Studies testing the information degradation hypothesis may help elucidate these issues.

\section{Information degradation hypothesis in visual search}

Recent studies testing the information degradation hypothesis have used visual search paradigms. Toner et al. (2012) administered to older and younger adults a touch-screen, digit cancellation task, in which participants had $60 \mathrm{~s}$ to cancel the $4 \mathrm{~s}$ and $9 \mathrm{~s}$ presented in a matrix of numbers ranging from 1 to 9 by physically touching the numbers on the screen (This study also reported results for Alzheimer's and Parkinson's disease patients, not discussed here.). The digit cancellation task was presented under three visual contrast levels - low (22\% Michelson contrast [(maximum - minimum luminance)/(maximum + minimum luminance)]), high (69\% Michelson contrast), and a unique, proximal match condition. The proximal match condition was designed to be a "vision-fair" condition, in which before completing the digit cancellation tasks, participants completed a backward masking, number identification task with a verbal response. For each participant, a unique identification accuracy threshold (i.e., critical contrast) was obtained from the contrast at which participants identified the numbers at $80 \%$ accuracy. As expected, the critical contrast threshold was higher for older than younger adults, representing an age-related decline in visual contrast sensitivity, and the older adults exhibited worse task performance than the younger adults in both the low and high visual contrast conditions. Consistent with the information degradation hypothesis, both the older and younger adults exhibited better task performance (in terms of the number of targets cancelled in the allotted time) in the high than low contrast condition. There was, however, no Age Group by Contrast Condition interaction, indicating that both the older and younger adults were equally affected by the contrast manipulation. The lack of an interaction may due to several reasons; for example, the younger adults may have been at a ceiling level of performance, or the screen-touch outcome measure, involving a manual response, may have masked effects of visual sensory processing. Intriguingly, for the analyses restricted to the vision-fair, proximal match condition, both the older and younger adults demonstrated comparable task performance. This study provides one of the most compelling pieces of evidence supporting the information degradation hypothesis, because not only were both the older and younger adults' task performance better at higher than lower visual contrast conditions, but the older adults' performance could be raised to the 
level of the younger adults by simply equating the proximal signal strength of the two age groups. The latter result demonstrates that age differences in task performance in the high and low visual contrast conditions were due to perceptual signal strength differences, rather than higher-order cognitive or motor/speed processing differences, that when equated led to equal task performance in both age groups.

In another study testing the information degradation hypothesis in visual search (Laudate et al., 2012), older and younger adults played bingo on a touch-screen computer presented under several visual conditions (This study also reported results for Alzheimer's and Parkinson's disease patients, not discussed here.). In order to ensure that the results were not due to age-differences in motor speed, the authors controlled for Purdue Pegboard (Lafayette Instrument, Lafayette, IL, United States) performance when appropriate. Across all conditions, the older adults exhibited slower responses than the younger adults. In one manipulation, participants played bingo with cards using either small or large type, which was essentially a manipulation of visual acuity. Consistent with the information degradation hypothesis, both the older and younger adults exhibited faster responses when playing the large compared to small type bingo cards. There was, however, no Age Group by Type Size interaction, indicating that both the older and younger adults were equally affected by the type size manipulation; similar to Toner et al. (2012), the lack of an interaction may be due to ceiling effects, where the task was not difficult enough to reveal age-sensitive effects. In another set of manipulations, participants played bingo with normal (62\% Michelson contrast) and enhanced ( $85 \%$ Michelson contrast) contrast cards. Neither the younger nor older adults were affected by the contrast manipulation, which differs from Toner et al. (2012) who found an effect of contrast. This difference between studies may be due to Laudate et al. (2012) using a higher baseline visual contrast (62\% Michelson contrast) than Toner et al. (22\% Michelson contrast). However, overall Laudate et al. supports the information degradation hypothesis, in which a manipulation of visual acuity affected both older and younger adults' task performance.

In summary, Toner et al. (2012) and Laudate et al. (2012) provide some support for the information degradation hypothesis, because simple visual manipulations affected task performance for both younger and older adults, and older adults' performance could be raised to the level of younger adults by equating the proximal signal strength of the two age groups (Toner et al., 2012). However, inconsistent with this hypothesis, both studies found that both the younger and older adults were equally affected by the contrast manipulations. As noted previously, the experiments may not have used sufficiently sensitive methods to elicit the predicted interaction.

\section{Information degradation hypothesis in other cognitive processes}

A few studies tested the information degradation hypothesis in other cognitive processes; however, these studies only included younger and not older adults. Ben-David and Schneider (2010) administered a Stroop task to a group of younger adults and manipulated the color contrast of the task stimuli - high-saturation and low-saturation; the low-saturation condition was designed to mimic deficient color perception observed in older adults. They found that the low-saturation condition increased Stroop interference effects, similar to effects typically observed within older adults; in a control condition, in which participants read words printed in a color-neutral contrast (e.g., "GREEN" printed white on black), the color contrast manipulation did not affect reaction times, allowing for greater specificity in the interpretation of the former result. The authors suggested that increased Stroop interference effects in older adults may be due to color perception deficiencies rather than inhibitory control deficiencies as commonly interpreted. However, because this study did not include older adults, these findings do not necessarily contradict traditional, Stroop interference effect interpretations in aging (e.g., Spieler et al., 1996).

Another study with younger adults (Gilmore et al., 2006) investigated whether stimulus contrast would affect performance on a modified version of the Digit-Symbol Substitution Test of the Wechsler Adult Intelligence Scale (WAIS; Wechsler, 1958) with three levels of difficulty, defined by increasing complexity levels of the symbols to be matched. This study included normal and degraded contrast groups. For the normal contrast group, the task stimuli contrast matched the visual contrast normally presented in the WAIS. For the degraded contrast group, the task stimuli contrast was degraded to resemble visual contrast sensitivity deficiencies typically observed in older adults by applying a digital filter to the normal contrast stimuli. The digital filter was constructed based upon the ratio of the spatial contrast sensitivity function of 20- and 80 -years-olds from a previous study (Owsley et al., 1983), in which the ratio was weighted to imitate the reduction of spatial frequency contrast observed within the 80 -years-olds. The authors found that the degraded contrast group made fewer correct substitutions than the normal contrast group on the easy and medium levels of difficulty. For the hardest level of the task, however, the degraded and normal contrast groups made a similar number of correct substitutions, but performance was still above chance, indicating that the results of this study were not due to the degraded stimuli being too difficult to see; the authors interpreted the lack of a group difference specifically within the most difficult condition as reflecting each contrast group requiring the use of a detailed serial search of the items because of the confusability of the symbols within this condition. Intriguingly, these findings match the results of a previous experiment conducted by the authors, in which younger and older adults completed this task, indicating that the visual contrast manipulation imitated age-effects. Furthermore, additional conditions determined that the described results were not due to simple differences in encoding speed for features, but rather were due to the visual confusability of the symbols. Consistent with the information degradation hypothesis, this indicates that degradation specifically affected higher-order cognitive processes. In summary, these results again demonstrate that even younger adults' task performance can be detrimentally affected if the visual contrast of task stimuli are manipulated to imitate visual perceptual deficiencies observed in older adults.

Lastly, Dickinson and Rabbitt (1991) examined the information degradation hypothesis in memory for short passages in a group of younger adults. In this study, participants read two short passages aloud while being recorded; one passage was read without any visual impairment and another was read in which the participants' vision was distorted with occlusion filter lenses. After reading the passages, participants were asked to recall content from the stories. During encoding, an analysis of the recordings showed that none of the participants made errors while reading in either condition, indicating that participants were able to successfully read both the distorted and non-distorted passages. During retrieval, the authors found evidence of visual perceptual signal strength affecting nonperceptual, higher-order cognitive processes, where participants recalled less content for the passages read under the distorted than non-distorted condition. This finding is especially important as it demonstrates that cognitive processes assessed during a task independent of the condition in which the perceptual manipulation occurred may still be affected by perceptual manipulations. In addition, the authors also found that participants with higher intelligence quotients (IQs) read passages under the distorted condition faster than participants with lower IQs. The authors inter- 
preted this finding as indicating that individuals with higher IQs may be better equipped with compensatory, top-down mechanisms allowing them to be less affected by degraded bottom-up signals.

\section{Information degradation hypothesis across multiple cognitive processes}

Although the previously described studies provide support for the information degradation hypothesis in individual cognitive processes, these experimental designs do not allow for a thorough examination of the extent of cognitive processes that are affected by direct manipulation of visual perceptual signal strength. This issue would be ideally examined in studies that contain a broad cognitive battery. In addition, testing this hypothesis with a broad cognitive battery may allow further evidence that findings are the result of perceptual manipulations influencing higher-order cognitive processes, as different cognitive processes may be differentially affected by identical perceptual manipulations. There have only been three studies, to our knowledge, that have taken this approach.

Anstey et al. (2006a) administered perceptual matching, digitsymbol matching, and associative memory tasks to older adults under low, medium, and high visual contrast conditions. Consistent with the information degradation hypothesis, the lower visual contrast conditions were associated with slower responses in all three tasks. In addition, the lowest visual contrast condition did not always have the longest reaction times, indicating that the results of this study were not due to simply making the stimuli too difficult to see and that the bottom-up, perceptual signals were interacting with higher-order cognitive processes rather than processes not of interest, such as motor speed; however, it should be noted that a limitation of this study is that the authors did not control for nuisance variables, such as motor speed, in order to further confirm that the described effects were not the result of the visual perceptual manipulations affecting these nuisance variables. These results demonstrate the validity of the information degradation hypothesis across three cognitive tasks, where visual contrast affected task performance across both perceptual (perceptual matching task) and higher-order cognitive tasks (digit-symbol matching and associative memory tasks).

Cronin-Golomb et al. (2007) also tested the information degradation hypothesis with multiple cognitive tasks: Younger and older adults completed cognitive tasks assessing letter and word identification, picture naming, spatial reasoning, and discrimination of unfamiliar faces (This study also reported results for Alzheimer's disease patients, not discussed here.). The stimuli for each task were presented at three contrast levels - low, medium, and high. The authors found that during the lower visual contrast conditions, both the older and younger adults exhibited slower responses for the letter and word reading tasks, and lower task accuracy for the picture naming tasks. In addition, consistent with the information degradation hypothesis, within each of these tasks, there were Age Group by Contrast Manipulation interactions, such that the older compared to younger adults exhibited a greater decrease in task performance when comparing higher to lower contrast conditions. It should be noted that in the word reading and picture naming tasks the low contrast condition did not yield the worst task performance, indicating that the results were not due to making the stimuli too difficult to see and demonstrate that bottom-up, perceptual signals differently interact with top-down processes of different cognitive states. Lastly, on the spatial reasoning and discrimination of unfamiliar faces tasks, the visual contrast conditions did not affect performance for either age group. However, the authors stated that this negative result may indicate that the visual contrast manipulations were not appropriate for the task stimuli due to the complexity of the stimuli in these tasks, as when processing complex stimuli, spatial frequency channel interactions may enhance or mask the visual signal (Thomas and Olzak, 2001). In summary, this study demonstrated that visual contrast may affect performance on several cognitive tasks, such as the letter reading, word reading and picture naming tasks, especially for older adults, whereas visual contrast may not affect other cognitive tasks, such as the spatial reasoning and discrimination of unfamiliar faces tasks. As all of the cognitive processes affected by the contrast manipulation decline with age (Baracat and Marquié., 1992; Madden, $1989,1992)$, these results suggest that a decline in visual contrast sensitivity may account for age-related decline in these cognitive processes.

Lastly, Bertone et al. (2007) tested the information degradation hypothesis within a group of younger adults across multiple cognitive domains. Occlusion filter lenses were used to modify participants' visual acuity, where participants were randomly assigned to one of three visual acuity groups - 20/20 (control condition), $20 / 40$, or $20 / 60$ visual acuity. After being assigned to one of the three groups, participants completed a broad range of cognitive tasks. Consistent with the information degradation hypothesis, participants in the lower visual acuity groups performed worse on tests designed to assess executive function, perceptual reasoning, visual search, and processing speed. Therefore, unlike Cronin-Golomb et al. (2007), Bertone et al. found that even higher-order processes, such as executive function and perceptual reasoning, were affected by visual degradation, suggesting that visual signal strength may affect higher-order cognitive processes. In addition, similar to two studies previously mentioned (Anstey et al., 2006a; Cronin-Golomb et al., 2007), the lowest visual acuity group (20/60 visual acuity) did not have the worst task performance in several of the tasks, indicating that the results were not related simply to the overall visual quality.

\section{Evidence inconsistent with the information degradation hypothesis}

Even though there are several studies supporting the information degradation hypothesis, there are studies inconsistent with this hypothesis. For example, Lindenberger et al. (2001) degraded older adults' visual acuity by having participants wear eyeglasses with occlusion filter lenses; the control participants wore glasses without the occlusion filter lenses. The authors assessed cognition with a broad cognitive battery and did not detect any differences in cognitive performance between older adults in the degraded and control groups. This study suggests that one of the alternative hypotheses may better explain the relation between visual perceptual and cognitive decline in aging (common-cause, sensory deprivation, cognitive load on perception), but the reason for these negative results may be that only visual acuity was manipulated. Modification of the participants' visual contrast sensitivity may have resulted in positive effects, as visual contrast sensitivity has been demonstrated to be more closely linked to cognition than visual acuity (Skeel et al., 2006; but cf. Madden and Greene, 1987).

There is also a literature examining the effects of cataract surgery on cognition in older adults, testing an important implication of the information degradation hypothesis, specifically that older adults' cognitive abilities should increase post-surgery. Unfortunately, the majority of these studies employed experimental designs that do not allow for definitive conclusions of the effects of cataract surgery on cognition. Typically, studies have either not included a control, non-surgery cataract group (Gray et al., 2006; Ishii et al., 2008; Jefferis et al., 2015), not randomized participants to either the control or surgery group (Hall et al., 2005; Tamura et al., 
2004), or were cross-sectional, epidemiological studies (Grodstein et al., 2003). The only study, to our knowledge, that included a randomized, control design did not detect a post-surgery improvement in cognitive performance, relative to the non-surgery, control group (Anstey et al., 2006b). Further replication with experimental designs similar to Anstey et al. (2006b) are necessary to determine whether cataract surgery may or may not improve cognitive performance in patients with cataracts.

\section{Conclusions and future directions}

In summary, there is preliminary support for the information degradation hypothesis, because several studies demonstrated that manipulation of the visual input quality directly affects both older and younger adults' task performance. These findings contradict assumptions of the common-cause, sensory deprivation, and cognitive load on perception hypotheses, which imply that manipulating perceptual signal strength should either not affect or not immediately affect cognitive performance. Even though there are studies supporting the information degradation hypothesis, this literature still contains four main limitations.

First, although several studies have found that older adults' cognitive performance is affected by visual perceptual signal strength, it is unclear if these effects are age-sensitive. Currently there are only three studies that have tested the information degradation hypothesis for both older and younger adults. One study found that older adults were more affected by visual manipulations (CroninGolomb et al., 2007), whereas the other two found that both older and younger adults were equally affected by visual manipulations (Laudate et al., 2012; Toner et al., 2012). As previously mentioned, the lack of age by visual manipulation interactions in Toner et al. (2012) and Laudate et al. (2012) may be due to ceiling effects, but future studies should continue to include study samples of both older and younger adults to test for age-sensitive effects. To further examine these effects, it would also be advantageous to include tasks with multiple levels of perceptual difficulty, to help distinguish age-specific effects from overall task complexity.

Second, it remains unclear if certain principles of the information degradation hypothesis are only applicable to adults. Although the information degradation hypothesis was originally created to describe the relation between cognitive and perceptual decline in healthy aging in adults, some of the principles of this hypothesis may apply to children with certain clinical disorders. For example, children with phenylketonuria (PKU), an inborn metabolism disorder, exhibit not only cognitive dysfunction (Diamond et al., 1997; Stemerdink et al., 1999; Welsh et al., 1990) but also decreased visual contrast sensitivity (Diamond and Herzberg, 1996; Gramer et al., 2013) relative to healthy children. It may be that decreased visual contrast sensitivity in children with PKU and other clinical disorders could account for some of the cognitive deficiencies present in these children; however, this has yet to be determined.

Third, it remains unclear if the information degradation hypothesis generalizes to all cognitive processes, as it has only been tested in a limited number of cognitive processes, typically tasks emphasizing visual search and comparison processes. Future studies should test the information degradation hypothesis in other cognitive processes that decline with age, such as different types of executive function (Verhaeghen and Cerella, 2002; Zelazo et al., 2004). In addition, more studies should include tasks representing a range of cognitive processes, similar to the experimental design of Cronin-Golomb et al. (2007). Single studies with a broad cognitive battery may further indicate if the information degradation hypothesis is specific to certain cognitive processes, and may provide further confidence that findings are the result of perceptual manipulations influencing higher-order cognitive processes, as different cognitive processes may be differentially affected by identical perceptual manipulations.

Fourth, the neural mechanisms contributing to the information degradation hypothesis remain unknown. Previous evidence suggests that older adults may engage compensatory neural processes in response to degraded perceptual inputs (Davis et al., 2008), but it remains unknown (a) how the scaled response to experimental manipulations of perceptual degradation affects compensatory neural processes; and (b) across cognitive domains, the extent of compensatory neural processes affected by perceptual degradation (Cabeza and Dennis, 2012; Grady, 2012; Park and Reuter-Lorenz, 2009). This work may help explain how certain aspects of cognition exhibit relatively little age-related decline, such as implicit memory, semantic processing and top-down attention (Burke et al., 2008; Howard and Howard, 2013; Madden, 2007).

Although not directly examining the neural mechanisms underlying the information degradation hypothesis, two recent studies have examined the relation between visual acuity and eventrelated potentials (ERPs) during a visual attention task in healthy older and young adults (Daffner et al., 2013; Porto et al., 2016). Both studies found that after controlling for visual acuity, some of the age-related differences in ERPs were no longer statistically different. These findings suggest that visual perceptual signal strength may affect neural mechanism differences underlying cognition between older and younger adults, such that the previously observed ERP, age differences may represent neural mechanisms compensating for degraded perceptual signals in older adults. Even though Porto et al. (2016) and Daffner et al. (2013) did not directly manipulate the visual perceptual signal of the participants, not allowing for a direct assessment of the neural mechanisms contributing to the information degradation hypothesis, other studies may take a similar approach to examine the neural substrates associated with the interaction between higher-order cognition and visual perception.

In summary, further testing the information degradation hypothesis may lead to a better understanding of age-related cognitive decline. From a clinical perspective, evidence supporting the information degradation hypothesis may have clear, practical clinical implications, by leading to interventions for enhancing older adults' perceptual signal strength (e.g., cataract surgery) in order to ameliorate age-related cognitive deficiencies. Further work in this area of research will hopefully elucidate mechanisms contributing to this decline and lead to the development of interventions designed to ameliorate and/or prevent cognitive deficiencies in older adults.

\section{Acknowledgements}

This research was supported by the National Institutes of Health research grantR01-AG039684 (DJM). The funding agency had no role in the decision to publish or preparation of the manuscript. The authors do not have any conflicts of interest to report.

\section{References}

Anstey, K.J., Butterworth, P., Borzycki, M., Andrews, S., 2006a. Between-and within-individual effects of visual contrast sensitivity on perceptual matching, processing speed, and associative memory in older adults. Gerontology 52, $124-130$.

Anstey, K.J., Lord, S.R., Hennessy, M., Mitchell, P., Mill, K., Von Sanden, C., 2006b. The effect of cataract surgery on neuropsychological test performance: a randomized controlled trial. J. Int. Neuropsychol. Soc. 12, 632-639.

Baltes, P.B., Lindenberger, U., 1997. Emergence of a powerful connection between sensory and cognitive functions across the adult life span: a new window to the study of cognitive aging? Psychol. Aging 12, 12.

Baracat, B., MarquiÉ, J.C., 1992. Age differences in sensitivity, response bias, and reaction time on a visual discrimination task. Exp. Aging Res. 18, 59-66.

Ben-David, B.M., Schneider, B.A., 2010. A sensory origin for color-word stroop effects in aging: simulating age-related changes in color-vision mimics 
age-related changes in stroop. Aging, Neuropsychology, and Cognition 17 $730-746$.

Bertone, A., Bettinelli, L., Faubert, J., 2007. The impact of blurred vision on cognitive assessment. J. Clin. Exp. Neuropsychol. 29, 467-476.

Buckner, R.L., Snyder, A.Z., Sanders, A.L., Raichle, M.E., Morris, J.C., 2000. Functional brain imaging of young, nondemented, and demented older adults. J. Cogn. Neurosci. 12, 24-34.

Burke, D.M., Shafto, M.A., Craik, F., Salthouse, T., 2008. Language and aging. In: Craik, F.I.M., Salthouse, T.A. (Eds.), The Handbook of Aging and Cognition. Third Ed. Taylor \& Francis, pp. 373-443.

Cabeza, R., Dennis, N.A., 2012. Frontal lobes and aging: deterioration and compensation. In: Stuss, D.T., Knight, R.T. (Eds.), Principles of Frontal Lobe Function, Second Edition. Oxford University Press New York, New York, United States, pp. 628-652.

Cronin-Golomb, A., Gilmore, G.C., Neargarder, S., Morrison, S.R., Laudate, T.M., 2007. Enhanced stimulus strength improves visual cognition in aging and Alzheimer's disease. Cortex 43, 952-966.

Daffner, K.R., Haring, A.E., Alperin, B.R., Zhuravleva, T.Y., Mott, K.K., Holcomb, P.J., 2013. The impact of visual acuity on age-related differences in neural markers of early visual processing. Neuroimage 67, 127-136.

Davis, S.W., Dennis, N.A., Daselaar, S.M., Fleck, M.S., Cabeza, R., 2008. Qué PASA? The posterior-anterior shift in aging. Cereb. Cortex 18, 1201-1209.

Diamond, A., Herzberg, C., 1996. Impaired sensitivity to visual contrast in children treated early and continuously for phenylketonuria. Brain 119, 523-538.

Diamond, A., Prevor, M.B., Callender, G., Druin, D.P., 1997. Prefrontal cortex cognitive deficitsn children treated early and continuously for PKU. Society for Research in Child Development 62 (i+ii + v+1-206).

Dickinson, C., Rabbitt, P., 1991. Simulated visual impairment: effects on text comprehension and reading speed. Clin. Vision Sci. 6, 301-308.

Evans, D.W., Ginsburg, A.P., 1985. Contrast sensitivity predicts age-related differences in highway-sign discriminability. Hum. Factors: J. Hum. Factors Ergon. Soc. 27, 637-642.

Faust, M.E., Balota, D.A., Spieler, D.H., Ferraro, F.R., 1999. Individual differences in information-processing rate and amount: implications for group differences in response latency. Psychol. Bull. 125, 777-799.

Gilmore, G.C., Spinks, R.A., Thomas, C.W., 2006. Age effects in coding tasks: componential analysis and test of the sensory deficit hypothesis. Psychol. Aging 21, 7-18.

Grady, C., 2012. The cognitive neuroscience of ageing. Nat. Rev. Neurosci. 13, 491-505.

Gramer, G., Förl, B., Springer, C., Weimer, P., Haege, G., Mackensen, F., Müller, E., Völcker, H.E., Hoffmann, G.F., Lindner, M., 2013. Visual functions in phenylketonuria-evaluating the dopamine and long-chain polyunsaturated fatty acids depletion hypotheses. Mol. Genet. Metab. 108, 1-7.

Gray, C.S., Karimova, G., Hildreth, A.J., Crabtree, L., Allen, D., O’Connell, J.E., 2006. Recovery of visual and functional disability following cataract surgery in older people: sunderland cataract Study. J. Cataract \& Refract. Surg. 32, 60-66.

Greene, H.A., Madden, D.J., 1987. Adult age differences in visual acuity, stereopsis, and contrast sensitivity. Optom. Vision Sci. 64, 749-753.

Grodstein, F., Chen, J., Hankinson, S.E., 2003. Cataract extraction and cognitive function in older women. Epidemiology 14, 493-497.

Hall, T.A., McGwin, G., Owsley, C., 2005. Effect of cataract surgery on cognitive function in older adults. J. Am. Geriatr. Soc. 53, 2140-2144.

Houston, J.R., Bennett, I.J., Allen, P.A., Madden, D.J., 2016. Visual acuity does not moderate effect sizes of higher-level cognitive tasks. Exp. Aging Res. 42, 221-263.

Howard, J.H., Howard, D.V., 2013. Aging mind and brain: is implicit learning spared in healthy aging? Front. Psychol. 4, 1-6.

Huettel, S.A., Singerman, J.D., McCarthy, G., 2001. The effects of aging upon the hemodynamic response measured by functional MRI. Neuroimage 13 , $161-175$.

Ishii, K., Kabata, T., Oshika, T., 2008. The impact of cataract surgery on cognitive impairment and depressive mental status in elderly patients. Am. J. Ophthalmol. 146, 404-409.

Jackson, G.R., Owsley, C., 2003. Visual dysfunction, neurodegenerative diseases, and aging. Neurol. Clin. North Am. 21, 709-728.

Jefferis, J.M., Clarke, M.P., Taylor, J.-P., 2015. Effect of cataract surgery on cognition, mood, and visual hallucinations in older adults. J. Cataract Refract. Surg. 41, 1241-1247

La Fleur, C.G., Salthouse, T.A., 2014. Out of sight, out of mind? Relations between visual acuity and cognition. Psychono. Bull. Rev. 21, 1202-1208.

Laudate, T.M., Neargarder, S., Dunne, T.E., Sullivan, K.D., Joshi, P., Gilmore, G.C., Riedel, T.M., Cronin-Golomb, A., 2012. Bingo! Externally supported performance intervention for deficient visual search in normal aging, Parkinson's disease, and Alzheimer's disease. Aging Neuropsychol. Cognit. 19, $102-121$.

Li, K.Z., Lindenberger, U., Freund, A.M., Baltes, P.B., 2001. Walking while memorizing: age-related differences in compensatory behavior. Psychol. Sci. $12,230-237$

Lin, F.R., Ferrucci, L., Metter, E.J., An, Y., Zonderman, A.B., Resnick, S.M., 2011. Hearing loss and cognition in the Baltimore Longitudinal Study of Aging. Neuropsychology 25, 763-770.

Lin, F.R., Yaffe, K., Xia, J., Xue, Q.-L., Harris, T.B., Purchase-Helzner, E., Satterfield, S. Ayonayon, H.N., Ferrucci, L., Simonsick, E.M., 2013. Hearing loss and cognitive decline in older adults. JAMA Intern. Med. 173, 293-299.
Lin, F., Ferrucci, L., An, Y., Goh, J., Doshi, J., Metter, E., Davatzikos, C., Kraut, M.A Resnick, S.M., 2014. Association of hearing impairment with brain volume changes in older adults. Neuroimage 90, 84-92.

Lindenberger, U., Baltes, P.B., 1994. Sensory functioning and intelligence in old age: a strong connection. Psychol. Aging 9, 339.

Lindenberger, U., Marsiske, M., Baltes, P.B., 2000. Memorizing while walking: increase in dual-task costs from young adulthood to old age. Psychol. Aging 15, 417.

Lindenberger, U., Scherer, H., Baltes, P.B., 2001. The strong connection between sensory and cognitive performance in old age: not due to sensory acuity reductions operating during cognitive assessment. Psychol. Aging 16, 196-205.

Madden, D.J., Greene, H.A., 1987. From retina to response: contrast sensitivity and memory retrieval during visual word recognition. Exp. Aging Res. 13, 15-21.

Madden, D.J., Whiting, W.L., Huettel, S.A., 2005. Age-related changes in neural activity during visual perception and attention. In: Cabeza, R., Nyberg, L., Park, D.C. (Eds.), Cognitive Neuroscience of Aging: Linking Cognitive and Cerebral Aging. Oxford University Press New York, New York, United States, pp. 157-185.

Madden, D.J., 1989. Visual word identification and age-related slowing. Cognit. Dev. 4, 1-29.

Madden, D.J., 1992. Four to ten milliseconds per year: age-related slowing of visual word identification. J. Gerontol. 47, P59-P68.

Madden, D.J., 2001. Speed and timing of behavioral processes. In: Birren, J.E., Schaie, K.W. (Eds.), Handbook of the Psychology of Aging. , fifth ed. Academic Press, San Diego, California, United States, pp. 288-312.

Madden, D.J., 2007. Aging and visual attention. Curr. Direct. Psycholo. Sci. 16, $70-74$.

Nameda, N., Kawara, T., Ohzu, H., 1989. Human visual spatio-temporal frequency performance as a function of age. Optom. Vision Sci. 66, 760-765.

Ng, E.H.N., Rudner, M., Lunner, T., Pedersen, M.S., Rönnberg, J., 2013. Effects of noise and working memory capacity on memory processing of speech for hearing-aid users. Int. J. Audiol. 52, 433-441.

Owsley, C., Sloane, M.E., 1987. Contrast sensitivity, acuity, and the perception of 'real-world' targets. Br. J. Ophthalmol. 71, 791-796.

Owsley, C., Sekuler, R., Siemsen, D., 1983. Contrast sensitivity throughout adulthood. Vision Res. 23, 689-699.

Owsley, C., 2011. Aging and vision. Vision Res. 51, 1610-1622.

Park, D.C., Reuter-Lorenz, P., 2009. The adaptive brain: aging and neurocognitive scaffolding. Annu, Rev. Psychol. 60, 173-196.

Pichora-Fuller, M.K., Schneider, B.A., Daneman, M., 1995. How young and old adults listen to and remember speech in noise. J. Acoust. Soc. Am. 97, 593-608.

Porto, F.H., Tusch, E.S., Fox, A.M., Alperin, B.R., Holcomb, P.J., Daffner, K.R., 2016. One of the most well-established age-related changes in neural activity disappears after controlling for visual acuity. Neuroimage 130, 115-122.

Raz, N., Lindenberger, U., Rodrigue, K.M., Kennedy, K.M., Head, D., Williamson, A. Dahle, C., Gerstorf, D., Acker, J.D., 2005. Regional brain changes in aging healthy adults: general trends, individual differences and modifiers. Cereb. Cortex 15, 1676-1689.

Ross, M., Yurgelun-Todd, D.A., Renshaw, P.F., Maas, L., Mendelson, J., Mello, N., Cohen, B., Levin, J., 1997. Age-related reduction in functional MRI response to photic stimulation. Neurology 48, 173-176.

Rubin, G.S., Roche, K.B., Prasada-Rao, P., Fried, L.P., 1994. Visual impairment and disability in older adults. Optom. Vision Sci. 71, 750-760.

Salthouse, T.A., 1996. The processing-speed theory of adult age differences in cognition. Psychol. Rev. 103, 403.

Salthouse, T.A., 2000. Aging and measures of processing speed. Biol. Psychol. 54, 35-54.

Schneider, B.A., Pichora-Fuller, M.K., 2000. Implications of perceptual deterioration for cognitive aging research. In: Craik, F.I.M., Ssalthouse, T.A. (Eds.), The Handbook of Aging and Cognition. , second ed. Lawrence Erlbaum Associates, Mahwah, New Jersey United States, pp. 155-219.

Scialfa, C.T., 2002. The role of sensory factors in cognitive aging research. Can. J. Exp. Psychol./Revue canadienne de psychologie expérimentale 56, 153-163.

Skeel, R.L., Nagra, A., VanVoorst, W., Olson, E., 2003. The relationship between performance-based visual acuity screening, self-reported visual acuity, and neuropsychological performance. Clin. Neuropsychol. 17, 129-136.

Skeel, R.L., Schutte, C., van Voorst, W., Nagra, A., 2006. The relationship between visual contrast sensitivity and neuropsychological performance in a healthy elderly sample. J. Clin. Exp. Neuropsychol. 28, 696-705.

Spieler, D.H., Balota, D.A., Faust, M.E., 1996. Stroop performance in healthy younger and older adults and in individuals with dementia of the Alzheimer's type. J. Exp. Psychol. Hum. Percept. Perform. 22, 461-479.

Stemerdink, N.B., van der Molen, M.W., Kalverboer, A.F., van der Meere, J.J., Huisman, J., de Jong, L.W., Slijper, F.M., Verkerk, P.H., van Spronsen, F.J., 1999. Prefrontal dysfunction in early and continuously treated phenylketonuria. Dev. Neuropsychol. 16, 29-57.

Tamura, H., Tsukamoto, H., Mukai, S., Kato, T., Minamoto, A., Ohno, Y., Yamashita H., Mishima, H.K., 2004. Improvement in cognitive impairment after cataract surgery in elderly patients. J. Cataract Refract. Surg. 30, 598-602.

Thomas, J.P., Olzak, L.A., 2001. Spatial phase sensitivity of mechanisms mediating discrimination of small orientation differences. J. Opt. Soc. Am. A 18, 2197-2203.

Tielsch, J.M., Sommer, A., Witt, K., Katz, J., Royall, R.M., 1990. Blindness and visual impairment in an American urban population: the Baltimore Eye SurveyArchives of Ophthalmology. Arch. Ophthalmol. 108, 286-290. 
Toner, C.K., Reese, B.E., Neargarder, S., Riedel, T.M., Gilmore, G.C., Cronin-Golomb, A., 2012. Vision-fair neuropsychological assessment in normal aging, Parkinson's disease and Alzheimer's disease. Psychol. Aging 27, 785.

Tun, P.A., McCoy, S., Wingfield, A., 2009. Aging, hearing acuity, and the attentional costs of effortful listening. Psychol. Aging 24, 761-766.

Verhaeghen, P., Cerella, J., 2002. Aging, executive control, and attention: a review of meta-analyses. Neurosci. Biobehav. Rev, 26, 849-857.

Ward, L.M., Aitchison, R.T., Tawse, M., Simmers, A.J., Shahani, U., 2015. Reduced haemodynamic response in the ageing visual cortex measured by absolute fNIRS. PLoS One 10, e0125012.

Weale, R.A., 1961. Retinal illumination and age. Light. Res. Technol. 26, 95-100.

Wechsler, D., 1958. The Measurement and Appraisal of Adult Intelligence. Williams \& Wilkins, Baltimore, Maryland, United States.
Welsh, M.C., Pennington, B.F., Ozonoff, S., Rouse, B., McCabe, E.R.B., 1990.

Neuropsychology of early-treated phenylketonuria: specific executive function deficits. Child Dev. 61, 1697-1713.

Wood, J.M., Owens, D.A., 2005. Standard measures of visual acuity do not predict drivers' recognition performance under day or night conditions. Optom. Vision Sci. 82, 698-705.

Zelazo, P.D., Craik, F.I., Booth, L., 2004. Executive function across the life span. Acta Psychol. 115, 167-183. 